\title{
A study of the phase instability of quasi-geostrophic Rossby waves on the infinite $\beta$-plane to zonal flow perturbations
}

\author{
L. Marié \\ Laboratoire de Physique des Océans, UMR6523 CNRS/IFREMER/IRD/UBO, IFREMER/Centre de Brest, \\ 29280 Plouzané, France
}

Received: 31 August 2009 - Revised: 2 December 2009 - Accepted: 7 December 2009 - Published: 2 February 2010

\begin{abstract}
The problem of the linear instability of quasigeostrophic Rossby waves to zonal flow perturbations is investigated on an infinite $\beta$-plane using a phase dynamics formalism. Equations governing the coupled evolutions of a zonal velocity perturbation and phase and amplitude perturbations of a finite-amplitude wave are obtained. The analysis is valid in the limit of infinitesimal, zonally invariant perturbation components, varying slowly in the meridional direction and with respect to time. In the case of a slow sinusoidal meridional variation of the perturbation components, analytical expressions for the perturbation growth rates are obtained, which are checked against numerical codes based on standard Floquet theory.
\end{abstract}

\section{Introduction}

There has been in recent years growing recognition of the existence in the mid-latitude atmosphere and oceans of the Earth as well as in the atmospheres of gaseous planets of large-scale persistent zonal jets (Galperin et al., 2004; Maximenko et al., 2008). These features have also been observed in a wide variety of numerical or analytical settings (Panetta, 1993; Vallis and Maltrud, 1993; Manfroi and Young, 1999; Nakano and Hasumi, 2005; Thomson and Young, 2007; Berloff et al., 2009; Dritschel and McIntyre, 2008), which seems to imply that their sustaining mechanism is extremely generic, and has minimal dependence on details of the flow configuration. One essential requirement seems however that a " $\beta$-effect" be present.

The appearance of zonal jets as end-products of $\beta$-plane turbulence has also been predicted long ago in the pioneering studies (Rhines, 1975, 1977), based on theoretical argu-

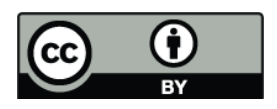

Correspondence to: L. Marié

(louis.marie@ifremer.fr) ments and numerical simulations. These studies have however left largely open the question of the actual mechanism responsible for the channeling of the flow energy to the zonal components.

Figure 1 sketches a conversion mechanism from waves to zonal flow, originally proposed as a Rossby wave instability mechanism by (Lorenz, 1972), and later mentioned as a possible forcing agency for jets by Manfroi and Young (1999). A finite-amplitude Rossby wave interacts on a $\beta$-plane with a zonal flow perturbation. The surfaces of constant phase move to the West. The zonal flow tends to distort them. At this point, it is easy to see that, the waves being transverse, fluid particles, whose velocity is parallel to the wavefronts, need to take a turn to the West, hence leave eastward momentum, when they cross the zonal flow perturbation. This continuous deposition of eastward momentum by the wave produces a positive feedback mechanism leading to exponential growth of the zonal perturbation. The $\beta$-effect, which at first seems not to play a key role in the mechanism, acts subtly as a "detuning" influence, in allowing the amplitude and phase perturbations to propagate at the group velocity associated to the wave, and non-zonal flow perturbations at their own phase velocity. Only in the case of zonal perturbations growing on purely meridional wave fronts does its influence vanish.

If quantitatively correct, this mechanism provides a very simple framework in which to interpret the growth and sustained existence of zonal jets. Indeed, very little requirements are imposed on the dynamical mechanisms responsible for the propagation or forcing of the waves. It seems actually possible that the same argument could be applied to a population of such waves with very generic spectral distribution.

A first consistency check of this idea is to try to use it in the study of the growth of zonal perturbations on one base wave. 


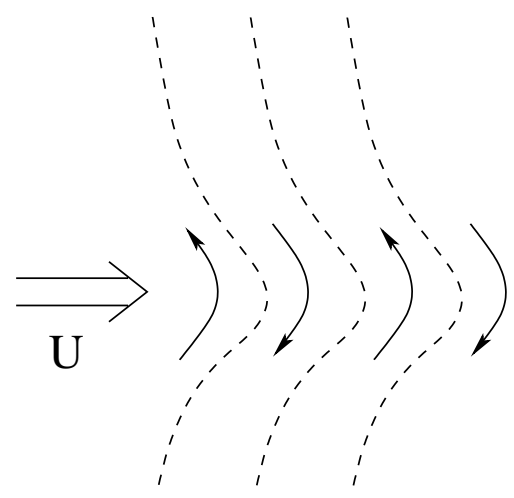

Fig. 1. Sketch of the Rossby wave instability mechanism. $U$ represents the zonal flow distribution. The dashed lines represent the wave fronts, and the full arrows represent the associated flow velocities.

The problem of the instability of a barotropic Rossby wave to perturbations on a $\beta$-plane has a long history. It has been in particular studied by (Lorenz, 1972; Gill, 1974; Sivashinsky, 1985), and in great detail by (Manfroi and Young, 2002) and (Lee and Smith, 2003). Many of these studies used truncated Floquet expansions, which renders analytical progress difficult.

Guided by the argument presented above, we have instead decided to consider the instability as the growth of a phase perturbation of the base pattern. This heuristic approach has the decisive advantage that the phase perturbation is uniform in the zonal direction, and that its evolution is governed by constant-coefficients equations. This simplifies the algebra considerably, and permits us to obtain relatively easily approximate analytical expressions for the growth rates, as well as to start investigating the (slightly) more realistic setting of the reduced-gravity quasi-geostrophic model.

In Sect. 2 we develop the phase dynamics formalism in the barotropic quasi-geostrophic dynamical framework. In Sect. 3 we extend it to the reduced-gravity quasi-geostrophic setting, in order to study the effect of the introduction of a stratification. In Sect. 4 we present a physical interpretation of the instability mechanism based on potential vorticity conservation, which is thus valid in stratified settings. We finally conclude and present possible directions for future work in Sect. 5.

\section{Barotropic case}

We study in this section the linear instability of a plane Rossby wave in the two-dimensional barotropic model. The setting is the same as that of (Gill, 1974), but we restrict ourselves to perturbations containing a purely zonal flow component. Our aim in this section is to work out an example of the phase-perturbation approach and show how it can ease the analysis with respect to the classical Floquet analysis.

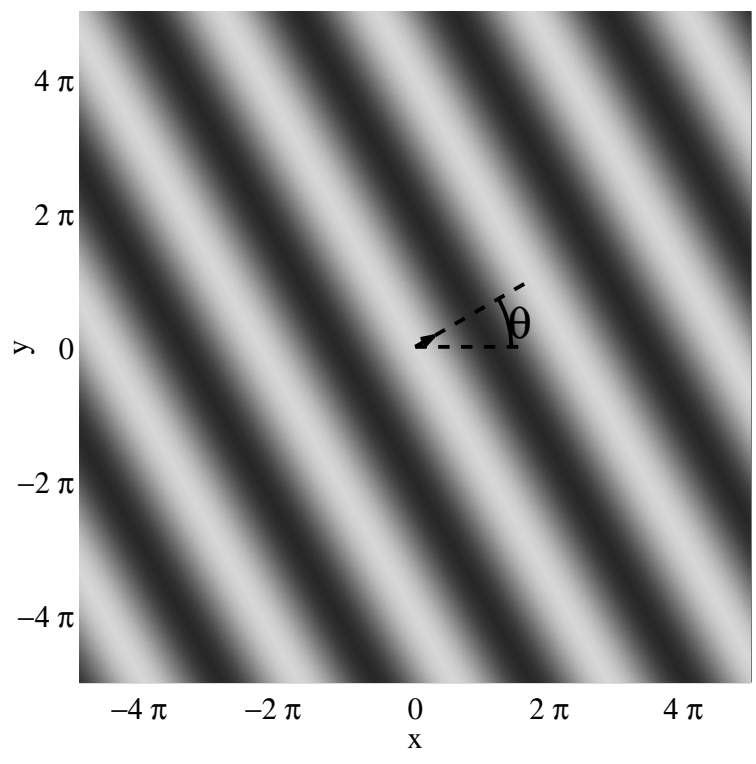

Fig. 2. Sketch of the base flow Rossby wave in nondimensional variables. The wavevector has unit length, and is at angle $\theta$ with respect to the zonal direction.

\subsection{Base flow and perturbations}

The flow whose stability we analyze in this section is a monochromatic Rossby wave, whose streamfunction is given by:

$\psi(x, y, t)=\operatorname{Re}\left(a e^{i[k x+l y-\omega t]}\right)$,

and whose dynamics on an infinite $\beta$-plane is governed by the two-dimensional barotropic vorticity conservation equation:

$\partial_{t} \Delta \psi+\beta \partial_{x} \psi+J(\psi, \Delta \psi)=0$,

where $J(f, g)=\partial_{x} f \partial_{y} g-\partial_{y} f \partial_{x} g$ denotes the Jacobian operator. We define $\theta=\operatorname{atan}(l / k)$ the angle the wavevector makes with the $\mathrm{x}$ direction, we choose as a length scale $1 / \sqrt{k^{2}+l^{2}}$, and as a time scale $\sqrt{k^{2}+l^{2}} / \beta$. With this choice of scales we obtain the governing equation in nondimensional form as:

$\partial_{t} \Delta \psi+\partial_{x} \psi+M J(\psi, \Delta \psi)=0$

and the base flow streamfunction, depicted in Fig. 2, as:

$\psi_{0}(x, y, t)=\operatorname{Re}\left(e^{i[\cos (\theta)(x+t)+\sin (\theta) y]}\right)$.

As in (Gill, 1974), the non-dimensional number controlling the effect of non-linearity, $M$, has been defined as:

$M=\frac{a\left(k^{2}+l^{2}\right)^{3 / 2}}{\beta}$.

It is easy to see that it is equal to the ratio of the velocity perturbation magnitude to the phase speed of the wave. 
As is well known, such a pure wave is an exact solution of the equation of motion for arbitrary values of $M$, but can be unstable to perturbations. The standard analysis using Floquet theory proceeds from there by linearizing the equation of motion around $\psi_{0}$, and studying the growth or decay rate of perturbations of the form

$\widetilde{\psi}(x, y, t)=e^{\lambda t} e^{i[K x+L y]} \widehat{\psi}(x, y)$,

with $\widehat{\psi}$ a function of period $2 \pi$ in the base flow wavevector direction. This course of action, which we have used to check the results of the phase dynamics analysis, is further pursued in Appendix A. The relation between the Floquet and the phase dynamics approaches is investigated in detail in Appendix C.

The insight provided by the instability mechanism alluded to above, though, entices us to introduce the perturbation in the form of slowly varying perturbations in the phase of the base flow Rossby wave. In order for the analysis to remain tractable, we perform a multiple-scales expansion and consider the phase perturbations to be small (of order $\varepsilon$ ) and to vary only in the y-direction, over large length scales of order $1 / \delta$. To account for the perturbation zonal mean velocity, we introduce a separate slowly-varying component of the total streamfunction, $\varepsilon \Psi(\delta y, t)$. The complete streamfunction finally reads

$$
\begin{aligned}
& \psi(x, y, t) \\
& =\varepsilon \Psi(\delta y, t) \\
& +\operatorname{Re}\left(e^{i[\cos (\theta)(x+t)+\sin (\theta) y+\varepsilon \phi(\delta y, t)+i \varepsilon \delta \chi(\delta y, t)]}\right)
\end{aligned}
$$

and the dynamical variables considered are $\phi, \chi$ and $U=$ $-\partial_{Y} \Psi$. In the following we denote as $Y$ the slow space variable $\delta y$. We emphasize that all three functions $\phi, \chi$ and $U$ are real. The influence of $\phi$, (resp. $\chi$ ) perturbations on the base flow stream function of Fig. 2 is depicted in Fig. 3a (resp. 3b).

\subsection{Zonal mean flow}

The equation of motion for the zonal flow perturbation can be obtained systematically by carrying the multiple-scales analysis to order $\varepsilon \delta^{3}$. This is rather tedious, and the end result can be obtained much more straightforwardly by considering directly the zonal average of Eq. (1). Denoting zonal averages by $<\cdot>$, we have:

$$
<\partial_{t} \Delta \psi>+<\partial_{x} \psi>+M<J(\psi, \Delta \psi)>=0
$$

Straightforward manipulations lead to the following equation for the zonally averaged component of the flow:

$\partial_{t y y} \Psi+M \partial_{y}<\partial_{x} \psi \cdot \partial_{y y} \psi>=0$

Supposing the forcing mechanism of the flow to be steady,
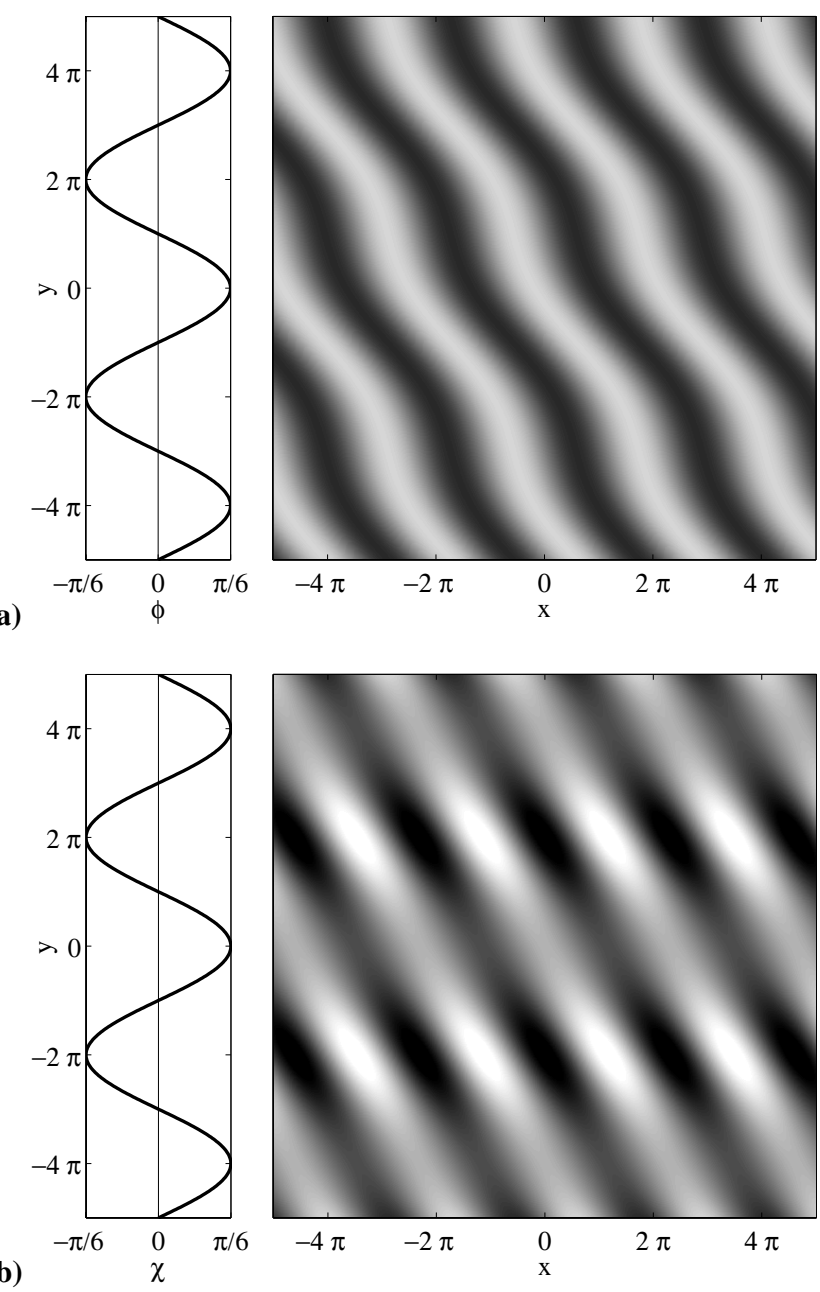

Fig. 3. Effect on the base flow Rossby wave of Fig. 2 of (a) a real phase anomaly $(\phi \neq 0$, the wave front shifts to the west for $\phi>0)$. (b) Imaginary phase anomaly $(\chi \neq 0$, the wave amplitude is low for $\chi>0)$.

we can integrate once with respect to $y$ to obtain:

$\partial_{t} U=M \partial_{y}<\partial_{x} \psi \cdot \partial_{y} \psi>=M<\partial_{x} \psi . \partial_{y y} \psi>$.

This equation expresses that the zonal mean flow is forced by convergence/divergence of the Reynolds tensions associated with the waves, or equivalently by meridional transport of relative vorticity across latitude circles. Clearly, only components of $\psi$ with the same dependencies on the fast variables as the base flow waves can produce Reynolds tensor components at order $\varepsilon$. 


\subsection{Multiple scales expansion, useful equalities}

At this point, we introduce the multiple time and space scales expansion

$\psi(x, y, t)$

$=\varepsilon \Psi\left(\delta y, t, \delta t, \delta^{2} t\right)$

$+\operatorname{Re}\left(e^{i\left[\cos (\theta)(x+t)+\sin (\theta) y+\varepsilon \phi\left(\delta y, t, \delta t, \delta^{2} t\right)+i \varepsilon \delta \chi\left(\delta y, t, \delta t, \delta^{2} t\right)\right]}\right)$

$+\varepsilon \delta \psi_{1}\left(x, y, \delta y, t, \delta t, \delta^{2} t\right)$

$+\varepsilon \delta^{2} \psi_{2}\left(x, y, \delta y, t, \delta t, \delta^{2} t\right)$.

This expression must be plugged into Eq. (1), and the systems obtained at order $\varepsilon$ (i.e. the linearized dynamics of infinitesimal perturbations) for increasing orders in $\delta$ are to be studied.

The following few identities are of frequent use in the computations:

- The fast spatial derivatives of all the quantities we manipulate satisfy:

$\partial_{t} f=\partial_{x} f, \partial_{y} f=\frac{\sin (\theta)}{\cos (\theta)} \partial_{x} f$.

- This entails that jacobians systematically expand as:

$J(f, g)=\delta\left[\partial_{x} f \partial_{Y} g-\partial_{x} g \partial_{Y} f\right]$.

- Finally,

$\Delta \psi_{0}=\left[-1+2 \delta \partial_{y Y}+\delta^{2} \partial_{Y Y}\right] \psi_{0}$,

- and obviously at all orders

$J(f, f)=0$.

\subsection{Low orders}

At order $\varepsilon$, Eq. (1) boils down to

$\partial_{t} \phi=0$.

This means that the phase perturbation depends on the slow time variables $\tau_{1}=\delta t$ and $\tau_{2}=\delta^{2} t$ only: the base flow Rossby wave satisfies the Rossby waves dispersion relation, and this absorbs all fast time-variations.

\subsection{Order $\varepsilon \delta$, phase evolution equation}

At order $\varepsilon \delta$, Eq. (1) reads:

$\mathcal{L} \psi_{1}$

$=-\partial_{t} \chi \psi_{0}$

$+\frac{1}{\cos (\theta)}\left[\partial_{\tau_{1}} \phi+\sin (2 \theta) \partial_{Y} \phi-M \cos (\theta) \partial_{Y} \Psi\right] \partial_{x} \psi_{0}$,

where $\mathcal{L}$ is the operator governing the linearized evolution of perturbations to the base flow, i.e.

$\mathcal{L} f=\partial_{t} \Delta f+\partial_{x} f+M J\left(\psi_{0},[\Delta+1] f\right)$,

where spatial derivatives are understood as taken in the fast space-variables only. It is quite easy to see that $\mathcal{L}$ is antihermitian (its terms contain only odd numbers of partial derivatives of $f$ ), and that the unperturbed streamfunction and its X-derivative are in its kernel. For Eq. (5) to be solvable for $\psi_{1}$, it is thus necessary that its right-hand side be orthogonal to $\psi_{0}$ and $\partial_{x} \psi_{0}$, which is only possible if

$\begin{aligned} \partial_{t} \chi & =0 \\ \partial_{\tau_{1}} \phi+\sin (2 \theta) \partial_{Y} \phi & =-M \cos (\theta) U\end{aligned}$

We thus obtain a first equation linking the evolution of the phase perturbation $\phi$ on the slow time scale $\tau_{1}$ with the zonal velocity perturbation $U$. It is easy to recognize on the lefthand side the propagation of a slowly varying perturbation of the "carrier" wave with the associated group velocity. The term on the right-hand side is also easily recognized as the phase perturbation due to the advection of wavefronts by the perturbation zonal mean velocity, one of the essential ingredients of the physical mechanism presented in Sect. 1. At this point, we see that imposing $\phi$ and $\chi$ to satisfy Eqs. (6) and (7) renders Eq. (5) autonomous. This means that Eq. (5) describes the linearized evolution of a free perturbation to $\psi_{0}$, i.e. the stability of $\psi_{0}$. It is for instance the starting point of the Floquet analysis of the problem, a path we have chosen not to follow. It is thus unnecessary to add as $\psi_{1}$ a new perturbation, and we set $\psi_{1}=0$.

\subsection{Order $\varepsilon \delta^{2}$, amplitude evolution equation}

Proceeding now to order $\varepsilon \delta^{2}$ in Eq. (1), we obtain:

$\mathcal{L} \psi_{2}$

$=-\partial_{t Y Y} \Psi$

$+\partial_{\tau_{2}} \phi \frac{1}{\cos (\theta)} \partial_{x} \psi_{0}$

$-\left[\partial_{\tau_{1}} \chi+\sin (2 \theta) \partial_{Y} \chi-2 \sin (\theta) \partial_{Y \tau_{1}} \phi-\cos (\theta) \partial_{Y Y} \phi\right] \psi_{0}$

$+2 M \sin (\theta) . \partial_{Y Y} \phi . \psi_{0} . \partial_{x} \psi_{0}$

Again, this equation must be solvable for $\psi_{2}$. It is easy from 
the definition of $\mathcal{L}$ to see that its kernel contains not only $\psi_{0}$ and $\partial_{x} \psi_{0}$ but also all the functions that are independent of $x$ and $y$. As $\mathcal{L}$ is anti-hermitian, we see that the solvability conditions read:

$$
\begin{aligned}
\partial_{t Y Y} \Psi & =0 \\
\partial_{\tau_{2}} \phi & =0 \\
\partial_{\tau_{1}} \chi+\sin (2 \theta) \partial_{Y} \chi & =2 \sin (\theta) \partial_{Y \tau_{1}} \phi+\cos (\theta) \partial_{Y Y} \phi
\end{aligned}
$$

The first of these equations imposes the time-evolution of the meridionally varying component of the zonal mean flow to take place on slow time scales. $\Psi$ still enjoys some freedom on the fast time scale, however, in that we are still free to add to $\Psi$ a component of the form $a(t) Y$, corresponding to fast changes in the uniform component of the zonal velocity. This is so because the vorticity Eq. (1), which we have chosen as the starting point of our study, filters out the uniform component of linear momentum. If this component of flow motion is of interest, as for instance in studies of its response to the growth of unstable disturbances, it is necessary to complement Eq. (1) with a second equation describing the coupling of the system with the forcing mechanism responsible for the basin-scale motion. Introducing such an equation provides a prescription for the time-variation of the uniform component of linear momentum, usually imposing it to occur on slow time scales. In the following we consider the system to be subject to time-independent forcing only, and set $\partial_{t} \Psi=0$ altogether.

The third of the equations describes the slow timevariation of $\chi$, the imaginary part of the phase perturbation, which describes local amplitude modulations in the carrier Rossby wave. We see that $\chi$ propagates meridionally with the relevant group velocity, and is coupled to the real part of the phase perturbation, $\phi$, through the source terms on the right-hand side. The first forcing term on the right-hand side is not unexpected: the equation we have obtained is describing the evolution of the wave amplitude. It is thus conceptually similar to the equations governing the Rossby waves activity density used in the study of "wave-mean flow interaction" (see e.g. Vallis, 2006). A consequence of these equations is that the mean squared potential vorticity (and not mean squared amplitude) present in wave packets is conserved during their interaction with a zonal flow (Young and Rhines, 1980). This implies that the product of the fourth power of the local wavevector length with the squared local amplitude flows at the group velocity, and is to be conserved at lowest order in our system: when $\partial_{Y} \phi$, which contributes to the wavevector, varies with time, the amplitude must vary simultaneously to keep the wave enstrophy density constant. The second term on the right-hand side originates in the divergence of the meridional component of the group velocity due to changes in latitude of $\partial_{Y} \phi$.
Replacing $\partial_{\tau_{1}} \phi$ by its expression obtained at order $\varepsilon \delta$, we obtain:

$$
\begin{aligned}
& \partial_{\tau_{1}} \chi+\sin (2 \theta) \partial_{Y} \chi \\
& =-M \sin (2 \theta) \partial_{Y} U+\cos (\theta)\left[1-4 \sin ^{2}(\theta)\right] \partial_{Y Y} \phi
\end{aligned}
$$

Equation (8) is this time not autonomous when we impose its right-hand side to satisfy the solvability conditions. It is clear from its right-hand side that the solution will vary with time and space as the product $\partial_{x} \psi_{0} . \psi_{0}$, i.e. twice as fast as $\psi_{0}$. As mentioned in Sect. 2.2, such components cannot contribute to the Reynolds tensor that drives the zonally averaged motion. Explicitly solving for $\psi_{2}$, though feasible, is thus not necessary.

\subsection{Zonal velocity perturbation evolution equation}

At this point, we are still lacking a prescription for the slow time-evolution of $U$, the zonal mean velocity. This last piece of information can be obtained by carrying the multiplescales analysis one order further in $\delta$. The same answer is however obtained much more easily by directly plugging the expression (4) in the equation of motion for the zonally averaged component of the flow (3), and using the knowledge about the solution gained at the previous orders. Keeping only terms of first order in $\varepsilon$ one has

$$
\begin{aligned}
\partial_{t} U= & M<\partial_{x} \psi \cdot \partial_{y y} \psi> \\
= & M<\partial_{x} \psi_{0} \cdot \partial_{y y} \psi_{0}> \\
= & M\left[-\sin ^{2}(\theta)+\varepsilon \delta 2 \sin (\theta) \partial_{Y} \phi\right]<\partial_{x} \psi_{0} . \psi_{0}> \\
& +M \varepsilon \delta^{2}\left[\frac{\partial_{Y Y} \phi}{\cos (\theta)}-\partial_{Y} \chi \frac{2 \sin (\theta)}{\cos (\theta)}\right]<\partial_{x} \psi_{0} . \partial_{x} \psi_{0}>
\end{aligned}
$$

Noting that products of terms whose fast spatial variations are in quadrature cannot contribute to a zonal mean, one finally obtains

$\partial_{\tau_{1}} U=\frac{M}{2}\left(\cos (\theta) \partial_{Y Y} \phi-\sin (2 \theta) \partial_{Y} \chi\right)$

We recognize in the first term of the right-hand side the curvature effect discussed in Sect. 1, and in the second term the effect of local attenuations in the amplitude of slanted waves. At this point, condensing the notation $\partial_{t}+\delta \partial_{\tau_{1}}+\ldots$ back to $\partial_{t}$, we can summarize the equations obtained for the timedependencies of $\phi, \chi$ and $\Psi$ as:

$$
\begin{aligned}
& \partial_{t} \phi=\delta[\left.-\sin (2 \theta) \partial_{Y} \phi-M \cos (\theta) U\right]+\mathcal{O}\left(\delta^{3}\right) \\
& \partial_{t} \chi=\delta\left[-\sin (2 \theta) \partial_{Y} \chi-M \sin (2 \theta) \partial_{Y} U\right. \\
& \\
&\left.\quad+\cos (\theta)\left[1-4 \sin ^{2}(\theta)\right] \partial_{Y Y} \phi\right]+\mathcal{O}\left(\delta^{3}\right) \\
& \partial_{t} U=\delta \frac{M}{2}\left[\cos (\theta) \partial_{Y Y} \phi-\sin (2 \theta) \partial_{Y} \chi\right]+\mathcal{O}\left(\delta^{2}\right)
\end{aligned}
$$




\subsection{Growth rates}

We introduce in Eq. (10) the following ansatz for the perturbation components:

$\left|\begin{array}{l}\phi \\ \chi \\ U\end{array}=e^{i[L Y-\Omega t]}\right| \begin{aligned} & \widehat{\phi} \\ & \widehat{\chi} \\ & \widehat{U}\end{aligned}$.

Equations (10) translate to the following eigensystem for $\widehat{\phi}$, $\widehat{\chi}, \widehat{U}$ and $\Omega$ :

$\frac{\Omega}{L} \mid \begin{aligned} & i \widehat{\phi} \\ & \widehat{\chi} / L \\ & \widehat{U} / L\end{aligned}$

$=\cos (\theta)\left[\begin{array}{lcr}2 \sin (\theta) & 0 & M \\ {\left[4 \sin ^{2}(\theta)-1\right]} & 2 \sin (\theta) & 2 M \sin (\theta) \\ -M / 2 & M \sin (\theta) & 0\end{array}\right] \times \mid \begin{aligned} & i \widehat{\phi} \\ & \widehat{\chi} / L \\ & \widehat{U} / L\end{aligned}$

$\Omega=0$ is an eigenvalue of system (11) for all values of $\theta$ (the physical meaning of this is at present unclear. It is actually true of the linearized equation of motion (A2), for zonal perturbations. The explanation is probably not straightforward, as the structure of the associated stationary mode is highly non-trivial). The remaining two eigenvalues of the system easily obtain as

$\Omega_{ \pm}=L\left[\sin (2 \theta) \pm i \frac{M \cos (\theta)}{\sqrt{2}} \sqrt{1-4 \sin ^{2}(\theta)}\right]$,

where the plus (resp. minus) sign corresponds to a growing (resp. decaying) perturbation.

A graph of the perturbation growth rate obtained with Eq. (12) for $M=0.5$ is presented in Fig. 4a, together with the equivalent result obtained numerically via high-order (Fig. 4b) and low-order (Fig. 4c) Floquet theory. We can see that in its region of applicability $(L \ll 1)$, Eq. (12) captures in a satisfactory way the dependence of the perturbation growth rate with respect to the perturbation wavenumber $L$ and the primary Rossby wave direction $\theta$. This proves that the phase perturbation approach provides a sound framework for the study of the problem at hand, and that it is worth extending to more complicated settings, as will be done in Sect. 3 .

Equation (12) predicts that the largest instability growth rates are obtained for $\theta=0^{\circ}$, i.e. for Rossby waves that have a nearly zonal wavevector. It also predicts the sharp transition at $\theta=30^{\circ}$ between instability $\left(\theta<30^{\circ}\right)$ and stability $\left(\theta>30^{\circ}\right)$, as well as the prefactor in the linear dependence of $\operatorname{Im}(\Omega)$ with respect to the perturbation wavenumber $L$ for small $L$. In the picture of the mechanism proposed in Sect. 1, the transition means that only Rossby waves whose wavevector make an angle below $30^{\circ}$ with the zonal direction can provide a significant source of energy to zonal jets, the most efficient being those with $\theta=0^{\circ}$. (Other Floquet results, not presented here, show that Rossby waves past $\theta=30^{\circ}$ are still unstable to long, but non-zonal, flow perturbations). The more complicated dependency of $\operatorname{Im}(\Omega)$ on
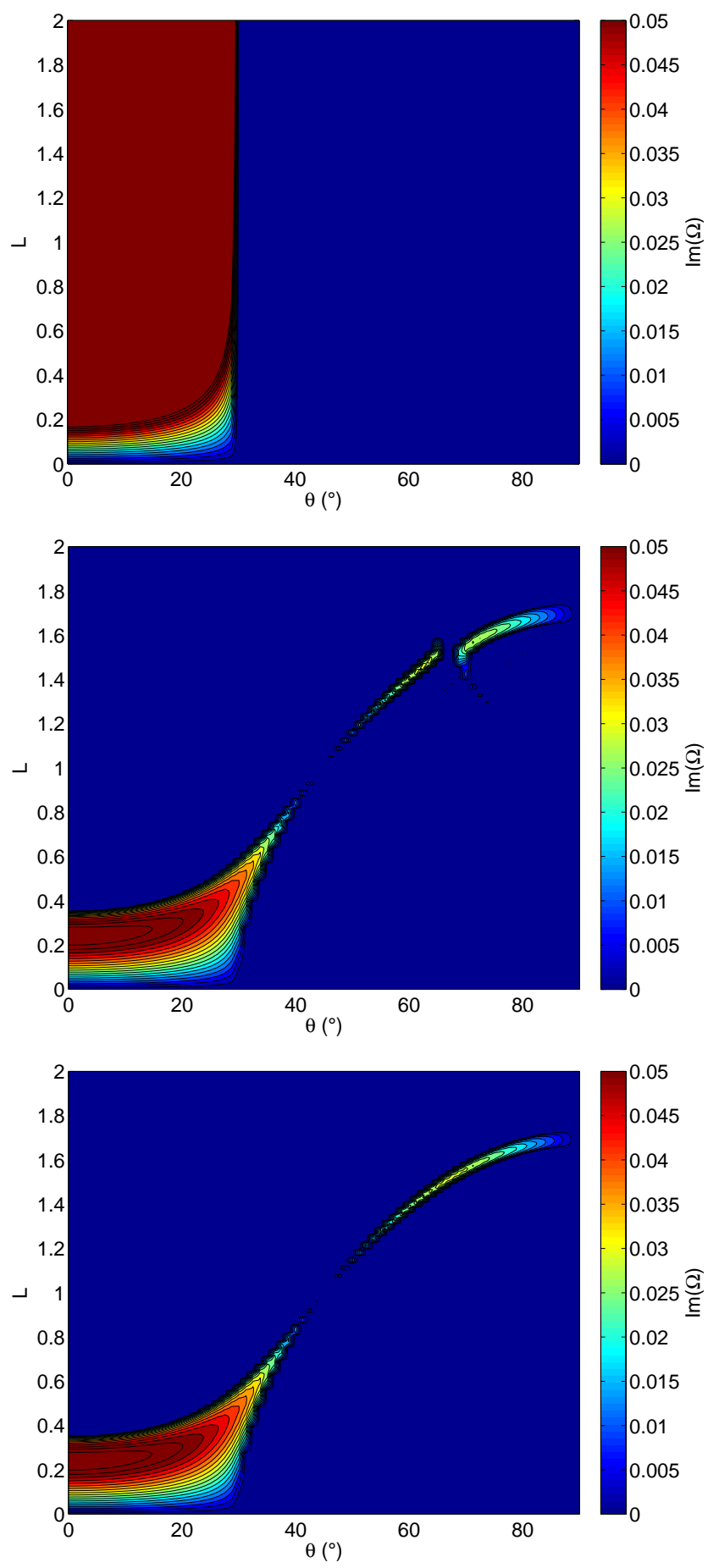

Fig. 4. Growth rates obtained for $M=0.5$, as a function of the primary Rossby wavevector direction $\theta$ and the meridional wavenumber of the phase perturbation $L$. (a) Analytical results obtained using Eq. (12). (b) Numerical results obtained with the Floquet code described in Appendix (A), with high-order expansion $(n \in$ $\{-16 \ldots 16\})$. (c) Numerical results obtained with the Floquet code with low-order expansion $(n \in\{-1,0,1\})$. 
$L$ and $\theta$ observed for larger values of $L$ remains out of reach, but seems of lesser oceanographic significance. Some of its features could certainly be reproduced by carrying the analysis leading to Eq. (12) to higher orders in $\delta$, but it is doubtful that a simple analytical expression for the growth rate such as Eq. (12) could be obtained.

The Floquet results extend the analytical expression (12) in an interesting way and provide insight into the behaviour of $\operatorname{Im}(\Omega)$ for large $L$. In particular, we see that the instability mechanism is scale-selective in that an optimal value of $L$ exists, for which the perturbation growth rate is maximal. The dependence of the optimal $L$ with respect to $M$ is studied below, but we observe that it remains of order 1, and that the highest growth rate is achieved for $\theta=0^{\circ}$, i.e. for purely meridional primary Rossby waves. Another remark is that past $\theta=30^{\circ}$, the base flow Rossby wave is no longer unstable to long-wavelength perturbations, as Eq. (12) indicates, but to perturbations whose wavenumber is more and more sharply selected as $\theta$ increases beyond $30^{\circ}$. The corresponding growth rates are clearly below optimal.

The dependence of the instability growth rate for $\theta=0^{\circ}$ with respect to $M$ and $L$ is shown in Fig. (5). We see that for small values of $M$ the range of unstable wavenumbers shrinks towards 0 , being bounded below by $L=0$ and above by $L=M / \sqrt{2}$. This indicates that the range of validity of the approximate expressions (10) also diminishes. At this point, however, we enter the range of validity of the approximate expression (18), which is valid in the small $M, L$ limit. Anticipating on the results of the following section, estimates of the optimal wavenumber $L^{*}$ and associated growth rate $\operatorname{Im}\left(\Omega^{*}\right)$ in the $M \leq 1$ range are:

$$
\begin{aligned}
L^{*} & =\frac{M}{2} \\
\operatorname{Im}\left(\Omega^{*}\right) & =\frac{M^{2}}{4}
\end{aligned}
$$

A final remark is that the low-order Floquet approach provides extremely precise growth rate estimates. The system can be solved analytically ( $\Omega=0$ is again a solution of the characteristic equation), but the expressions for the growth rates are cumbersome. The main motivation for the choice of the phase dynamics approach over its Floquet counterpart for the present study lies thus not in its accuracy, which is actually rather poor, but in the clear physical significance of the perturbation components $\phi, \chi, U$, and the insight their approximate equations of motion provide into the problem. The links between the phase-dynamics and low-order Floquet approaches are analyzed in Appendix C. There it is shown that a one-to-one mapping exists between the components of the $n=0, \pm 1$ Floquet perturbation and $\phi, \chi$ and $U$. The Floquet expansion however makes no assumptions regarding the scales of variations of the perturbations with respect to $y$, and yields more general equations of motion. The fact that it provides very accurate estimates of the growth rates shows that the instability indeed relies on phase and amplitude perturba-

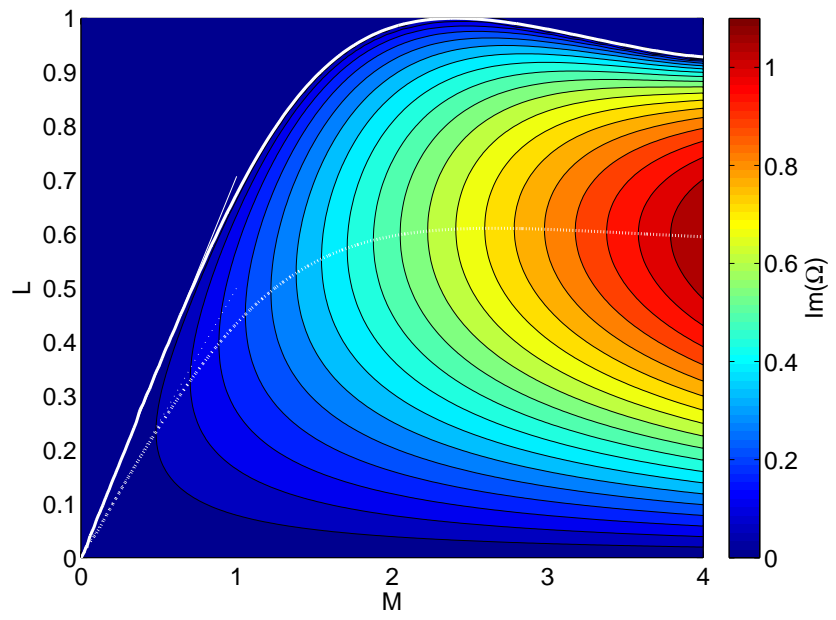

Fig. 5. Growth rates for $\theta=0$ as a function of $M$ and the perturbation wavenumber $L$, as obtained with the Floquet code. The full line marks the maximal value of $L$ for which instability is possible as a function of $M$. The dotted line marks the value of $L$ for which the instability growth rate is maximal as a function of $M$. The lines $L=\frac{M}{2}$ (optimal growth rate) and $L=\frac{M}{\sqrt{2}}$ (marginal stability line) have been marked to guide the eye.

tions to the base wave, and not on changes on its planform, such as needed by a "varicose" mode of instability, for instance. This finding actually carries over to the case of nonzonal perturbations (not shown).

\section{Quasi-geostrophic reduced gravity ("one and a half layer") case}

The phase dynamics approach has been applied to the study of mixes of barotropic and baroclinic waves in the quasigeostrophic two-layers model setting. This work is heavily computational, and will not be presented here for the sake of brevity. We will instead sketch the study of the reduced-gravity quasi-geostrophic "one and a half layer" model, which captures, with more acceptable conciseness, some of the major differences with the purely barotropic case (as for instance the introduction in the system of a length scale, the Rossby deformation radius, and the need to express the physical mechanism underlying the instability in terms of potential vorticity rather than linear momentum).

\subsection{Equations of motion}

In this section, the reduced-gravity quasi-geostrophic potential vorticity is defined as

$q=\Delta \psi-\frac{f^{2}}{g^{\prime} H} \psi$,

where $g^{\prime}$ is the reduced gravity corresponding to the interface bounding the active layer, and $H$ is the active layer rest 
thickness. We keep as a length scale the base flow wavelength divided by $2 \pi$, in order to have a unit modulus base flow wavevector. The non-dimensional potential vorticity then reads

$q=\Delta \psi-B \psi$,

where

$B=\frac{f^{2}}{g^{\prime} H\left(k^{2}+l^{2}\right)}$

is the inverse square of the non-dimensional Rossby deformation radius, and is a non-dimensional measure of stratification. Small (resp. large) $B$ values correspond to a small (resp. large) base flow wavelength with respect to the Rossby deformation radius.

We adapt the non-dimensionalization time scale so the phase velocity is 1 in non-dimensional units, i.e. we use as a time scale

$\tau=\frac{(1+B) \sqrt{k^{2}+l^{2}}}{\beta}$.

The non-dimensional measure of non-linearity then reads

$M=\frac{a\left(k^{2}+l^{2}\right)^{3 / 2}(1+B)}{\beta}$

and the quasi-geostrophic equation of motion (1) reads

$\partial_{t}[\Delta \psi-B \psi]+(B+1) \partial_{x} \psi+M J(\psi, \Delta \psi)$,

while the base flow streamfunction is again

$\psi_{0}(x, y, t)=\operatorname{Re}\left(e^{i[\cos (\theta)(x+t)+\sin (\theta) y]}\right)$.

The evolution equation for the zonal mean flow perturbation reads

$\partial_{t}\left[\partial_{y y}-B\right] \Psi+M \partial_{y}<\partial_{x} \psi \cdot \partial_{y y} \psi>=0$.

Due to the appearance of the vortex stretching term in the potential vorticity conservation equation, this equation cannot be straightforwardly transformed into one for $U$. This entails many differences in behaviour, which will be discussed as the analysis proceeds.

We finally use for $\psi$ the same multiple scales expansion as before:

$$
\begin{aligned}
& \psi(x, y, t) \\
& =\varepsilon \Psi\left(\delta y, t, \delta t, \delta^{2} t\right) \\
& +\operatorname{Re}\left(e^{i\left[\cos (\theta)(x+t)+\sin (\theta) y+\varepsilon \phi\left(\delta y, t, \delta t, \delta^{2} t\right)+i \varepsilon \delta \chi\left(\delta y, t, \delta t, \delta^{2} t\right)\right]}\right) \\
& +\varepsilon \delta \psi_{1}\left(x, y, \delta y, t, \delta t, \delta^{2} t\right) \\
& +\varepsilon \delta^{2} \psi_{2}\left(x, y, \delta y, t, \delta t, \delta^{2} t\right) .
\end{aligned}
$$

As before, the base flow streamfunction has been defined such that the equation of motion is trivially satisfied at or$\operatorname{der} \varepsilon \delta^{0}$.

\subsection{Order $\varepsilon \delta^{1}$, phase perturbation evolution equation}

At this order Eq. (14) reads:

$$
\begin{aligned}
0= & \mathcal{L}\left(\psi_{1}\right) \\
& -B \partial_{\tau_{1}} \Psi \\
& -\frac{\partial_{x} \psi_{0}}{\cos (\theta)}\left[(B+1) \partial_{\tau_{1}} \phi+\sin (2 \theta) \partial_{Y} \phi-M \cos (\theta) \partial_{Y} \Psi\right],
\end{aligned}
$$

where now

$\mathcal{L} f=\partial_{t}[\Delta-B] f+\partial_{x} f+M J\left(\psi_{0},[\Delta+1] f\right)$.

The $\mathcal{L}$ operator is as before anti-hermitian, possesses a similar kernel, and the solvability conditions are straightforwardly obtained as:

$$
\begin{aligned}
\partial_{\tau_{1}} \phi+\frac{\sin (2 \theta)}{B+1} \partial_{Y} \phi & =-\frac{M}{B+1} \cos (\theta) U \\
B \partial_{\tau_{1}} \Psi & =0
\end{aligned}
$$

The equation governing the evolution of the phase perturbation is just the transposition to the one and a half layer setting of that obtained in the barotropic case: the phase perturbation propagates with the group velocity associated to the carrier wave, and is forced by a Doppler effect term. This part of the analysis carries over just as straightforwardly to the twolayer and actually to the continuously stratified case, though in these cases all the normal mode components of the zonal velocity perturbations induce Doppler effect terms, which all possess different weighting factors. As regards the equation governing $\Psi$, we see that, in the $B \neq 0$ case (corresponding to a Rossby deformation wavelength not infinitely large with respect to the base flow wavelength), the vortex stretching term, which is the dominant part of the potential vorticity at the $Y$-dependence length scale, forbids the zonal flow perturbation to evolve on the first slow time scale $\tau_{1}$.

We solve the autonomous equation by choosing $\psi_{1}=0$. In more complicated settings such as the two-layer or the continuously stratified models, non-linear advection of potential vorticity perturbations in one baroclinic mode by the other mode velocities induces forced contributions at sum and difference frequencies, and with different vertical structures. In these cases, $\psi_{1}$ is different from zero, and must explicity be solved for. At the next order in the analysis, the forced components interact again with the original waves, forcing back resonant terms which appear in the solvability conditions for the $\varepsilon \delta^{2}$ problems. This two-stage process permits back and forth transfer of energy between modes of different vertical structures, and thus affects their amplitudes. It is thus expected that terms responsible for these exchanges occur in the order $\varepsilon \delta$ (creation of the forced contributions) and $\varepsilon \delta^{2}$ (back-effect on the free waves) expressions. Taking account of these effects renders the analysis heavily computational. 


\subsection{Order $\varepsilon \delta^{2}$, amplitude perturbation evolution equation}

At this order Eq. (14) reads:

$$
\begin{aligned}
0= & \mathcal{L}\left(\psi_{2}\right) \\
& -B \partial_{\tau_{2}} \Psi \\
& +\left[(B+1) \partial_{\tau_{1}} \chi+\sin (2 \theta) \partial_{Y} \chi-\cos (\theta) \partial_{Y Y} \phi\right. \\
& \left.-2 \sin (\theta) \partial_{\tau_{\tau_{1}}} \phi\right] \psi_{0} \\
& -\frac{(B+1)}{\cos (\theta)} \partial_{\tau_{2}} \phi . \partial_{x} \psi_{0} \\
& -2 M \sin (\theta) \partial_{Y Y} \phi . \psi_{0} . \partial_{x} \psi_{0} .
\end{aligned}
$$

This equation can be solved for $\psi_{2}$ if the following conditions are met:

$$
\begin{aligned}
B \partial_{\tau_{2}} \Psi & =0 \\
\partial_{\tau_{2}} \phi & =0 \\
\partial_{\tau_{1}} \chi+\frac{\sin (2 \theta)}{B+1} \partial_{Y} \chi & =2 \frac{\sin (\theta)}{B+1} \partial_{Y \tau_{1}} \phi+\frac{\cos (\theta)}{B+1} \partial_{Y Y} \phi
\end{aligned}
$$

The meridional vorticity flux divergence due to the amplitude and phase perturbations vanishes again at this order, which prevents an evolution of the vortex stretching associated to the zonal velocity perturbation. The third solvability condition governs the evolution of the wave amplitude perturbation. We recognize again in the right-hand side forcing terms caused by wave enstrophy density conservation. The first term serves the purpose of preventing wave enstrophy density variations due to changes in the local wavevector length, and the second of preventing variations due to changes in the local group velocity. Both these terms carry over trivially to the two-layer and the continuously stratified case. As mentioned above, though, other forcing terms corresponding to non-linear transfer of energy between the different baroclinic modes of the base flow wave mix appear in these cases. These terms scale as $M^{2}$, and could probably be neglected in a first approach to the problem.

In the present case, the remaining forcing terms are orthogonal to $\psi_{0}$ and $\partial_{x} \psi_{0}$, and can only force $\psi_{2}$ components with the same spatial and temporal frequencies. The product of $\psi_{2}$ with functions with the structure of $\psi_{0}$ and $\partial_{x} \psi_{0}$ can thus not have non-zero zonal mean, and will thus not be able to force the zonal flow perturbation. It is thus not necessary to solve for $\psi_{2}$ in the present reduced-gravity case. Once again, this part of the argument does not carry over to multiple vertical modes settings.

\subsection{Zonal perturbation evolution equation}

Again, we resort to the trick of plugging the expression of $\psi$ directly into the zonally averaged equation of motion (15), as a shortcut to pushing the multiple scales analysis to order $\varepsilon \delta^{3}$.

$$
\begin{aligned}
& \varepsilon \delta^{3} \partial_{\tau_{3}}\left[\delta^{2} \partial_{Y Y}-B\right] \Psi \\
& =-M \partial_{y}<\partial_{x} \psi \cdot \partial_{y y} \psi> \\
& =-M \partial_{y}<\partial_{x} \psi_{0} \cdot \partial_{y y} \psi_{0}> \\
& =-M \partial_{y}<\partial_{x} \psi_{0} \cdot\left[-\sin (\theta)^{2}-2 \varepsilon \delta \sin (\theta) \partial_{Y} \phi-\varepsilon \delta^{2} \partial_{Y Y} \chi\right] \psi_{0}> \\
& \quad-\varepsilon \delta^{2} \frac{M}{\cos (\theta)} \partial_{y}<\partial_{x} \psi_{0} \cdot\left[\partial_{Y Y} \phi-2 \sin (\theta) \partial_{Y} \chi\right] \partial_{x} \psi_{0}>
\end{aligned}
$$

Again, zonal averages can only contain contributions from products of terms whose spatial variations are in phase. We finally obtain:

$\partial_{\tau_{3}}\left[\delta^{2} \partial_{Y Y}-B\right] \Psi=\frac{M}{2}\left[\sin (2 \theta) \partial_{Y Y} \chi-\cos (\theta) \partial_{Y Y Y} \phi\right]$

Though it is not immediately apparent at first sight, this equation has a very different meaning from that obtained in the pure barotropic case, as can be readily observed by expressing it in terms of the zonal velocity perturbation. The dynamical variable dominating the dynamics of the zonal flow perturbation is now the vortex stretching component of the zonal flow potential vorticity. The growth of the zonal flow perturbation is dependent, not on a convergence of the linear momentum fluxes present in the base wave, which would force the order $\delta^{2}$ relative vorticity term on the left-hand side, but on a convergence of the potential vorticity fluxes. This difference is further discussed below. We recall here the equations of slow evolution of the Rossby wave phase and amplitude obtained in this section:

$$
\begin{aligned}
\partial_{t} \phi & =\delta\left[-\frac{\sin (2 \theta)}{(B+1)} \partial_{Y} \phi+M \frac{\cos (\theta)}{(B+1)} \partial_{Y} \Psi\right]+\mathcal{O}\left(\delta^{3}\right) \\
\partial_{t} \chi & \delta\left[-\frac{\sin (2 \theta)}{(B+1)} \partial_{Y} \chi+\frac{\cos (\theta)}{(B+1)^{2}}\left[B+1-4 \sin ^{2}(\theta)\right] \partial_{Y Y} \phi\right. \\
& \left.+M \frac{\sin (2 \theta)}{(B+1)^{2}} \partial_{Y Y} \Psi\right]+\mathcal{O}\left(\delta^{3}\right) \\
\partial_{t}\left[\delta^{2} \partial_{Y Y}-B\right] \Psi= & \delta^{3} \frac{M}{2}\left[\sin (2 \theta) \partial_{Y Y} \chi-\cos (\theta) \partial_{Y Y Y} \phi\right]+\mathcal{O}\left(\delta^{4}\right) .
\end{aligned}
$$

As is clearly apparent from the third equation, the system changes behaviour in the $B \rightarrow 0$ limit. This limit corresponds to the situation in which the Rossby radius of deformation becomes infinite with respect, not only of the base flow wavelength, but also with respect to the scale of slow $Y$-dependence. In this case all flow structures are governed by the requirement of conservation of relative vorticity, and the vortex stretching term becomes unimportant. This is the case we have studied in the previous section.

\subsection{Growth rates}

Again, we introduce in Eqs. (16) the following ansatz for the perturbation components:

$$
\left|\begin{array}{l}
\phi \\
\chi \\
\Psi
\end{array}=e^{i[L Y-\Omega t]}\right| \begin{aligned}
& \widehat{\phi} \\
& \widehat{\chi} \\
& \widehat{\Psi}
\end{aligned} .
$$


Equations (16) translate to the following eigensystem for $\widehat{\phi}$, $\widehat{\chi}, \widehat{U}$ and $\Omega$ :

$\Omega \mid \begin{aligned} & \widehat{\phi} \\ & i \widehat{\Psi}\end{aligned}$

$=L \frac{\cos (\theta)}{(B+1)}\left[\begin{array}{lcr}2 \sin (\theta) & 0 & -M \\ L\left[1-\frac{4 \sin ^{2}(\theta)}{(B+1)}\right] & 2 \sin (\theta) & 2 L M \frac{\sin (\theta)}{(B+1)} \\ L^{2} \frac{M(B+1)}{2\left(L^{2}+B\right)} & L \frac{M \sin (\theta)(B+1)}{\left(L^{2}+B\right)} & 0\end{array}\right]$

$\times \mid \begin{aligned} & \widehat{\phi} \\ & i \widehat{\chi} \\ & \widehat{\Psi}\end{aligned}$

Once again, $\Omega=0$ is an eigenvalue of the problem (the physical meaning of this has again not been investigated). The remaining two eigenvalues can be easily obtained as:

$\Omega_{ \pm}=\frac{L \sin (2 \theta)}{(B+1)} \pm i \frac{M L^{2} \cos (\theta)}{(B+1)} \sqrt{\frac{\left[B+1-4 \sin ^{2}(\theta)\right]}{2\left(B+L^{2}\right)}}$

Figure 6 displays the growth rates obtained with the Floquet numerical code in the $\theta=0^{\circ}$ case, for increasing values of $B$, i.e. for decreasing Rossby deformation radii measured with respect to the base flow wavelength. The main characteristics of these growth rates in the $L \rightarrow 0$ limit are well recovered by formula (18), except for the existence of a threshold value of $M$. A description of the behaviour near threshold can be obtained by setting $M$ to be of order $\delta^{2}$, and pushing the analysis one order further in $\delta$. The analysis in the general $\theta$ case is tedious. The $\theta=0^{\circ}$ special case is however fairly easy, and one obtains the frequency in the $M \rightarrow 0$, $L \rightarrow 0$ limit as

$\Omega_{\theta=0}=\frac{L^{2}}{(1+B)} \sqrt{1-\frac{M^{2}(B+1)}{2\left(B+L^{2}\right)}}$,

which agrees well with the Floquet results. The threshold value $M_{c}=\sqrt{\frac{2\left(B+L^{2}\right)}{(B+1)}}$ for instability in the $L \rightarrow 0$ limit agrees closely with the observed behaviour. Another interesting point apparent in Fig. 6 is the fact that the scale selection is essentially independent of $B$ and $M$ for $M \geq 2$. The most unstable perturbation has a non-dimensional wavenumber $L \simeq 0.7$ in all cases, corresponding to a meridional length scale roughly equal to 1.4 times the base wavelength. The introduction in the problem of an intrinsic length scale, the Rossby radius of deformation, seems to have a small influence in this respect. The length scale which dominates the scale selection pattern is clearly the base flow wavelength.

The introduction of the stratification, however, has a clear influence on the growth rates themselves, which have a marked decreasing tendency as $B$ rises (i.e. as the Rossby radius of deformation diminishes for a fixed base flow wavelength). This is consistent with the $B^{-1}$ scaling expected at large $M$ from formula (18). This decrease is genuine, in the sense that it persists even if the growth rate is examined in its
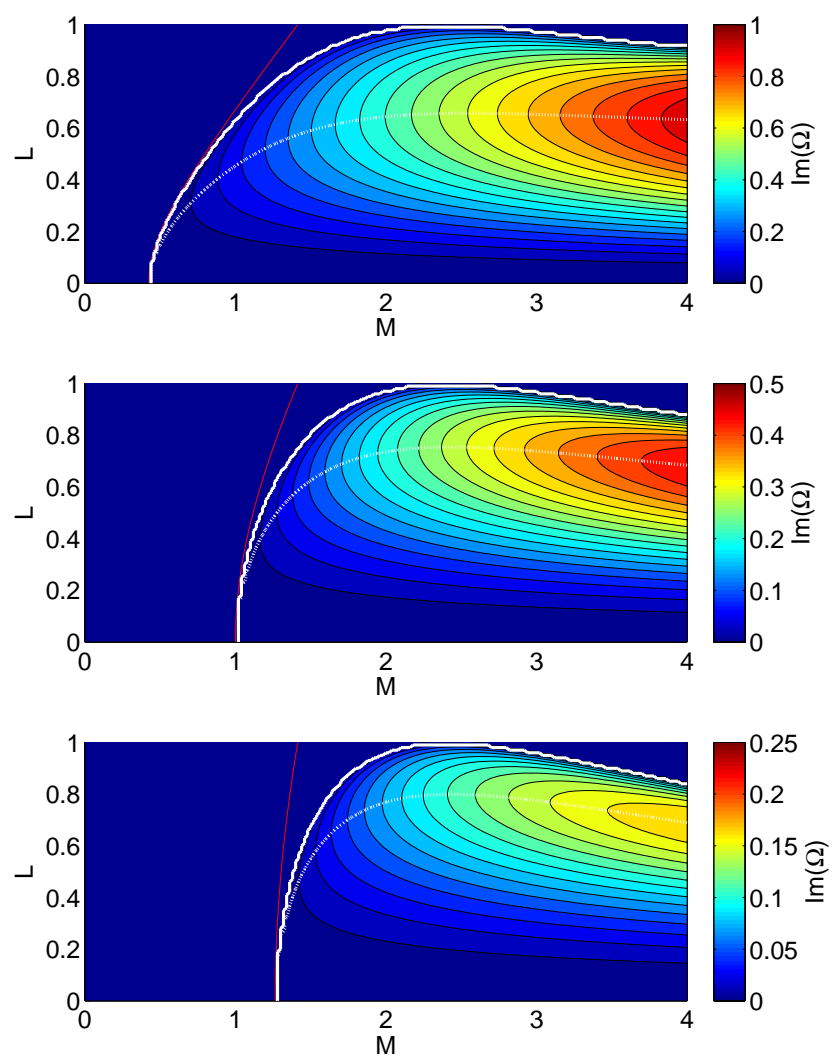

Fig. 6. Growth rates obtained using the Floquet numerical code in the reduced gravity quasi-geostrophic setting for $B=0.1$ (top), $B=1$ (center), $B=4$ (bottom), as a function of the non-linearity parameter $M$ and the perturbation meridional wavenumber $L$. The $\theta$ angle is held fixed to $0^{\circ}$. The full white line marks the limit of the unstable region of the parameter space. The dashed white line marks the maximum growth rate for fixed $M$. The thin red line marks the analytically calculated threshold value of $M$.

dimensional form (the time scale also increases with $B$, and the change of $M$ when $B$ varies for fixed base flow velocities is not sufficient to compensate).

Figure 7 compares the growth rates obtained analytically and with the Floquet code for $M=2$ and $B=0.1,1$ and 4 , as functions of the angle $\theta$ and the meridional perturbation wavenumber $L$. Figure 6 shows $M=2$ to be well above the instability threshold for $\theta=0^{\circ}$ for all these values of $B$. Once again, we see that the growth rates and their dependencies with respect to $\theta$ are satisfactorily captured by the approximate expression Eq. (18) for $L$ small. The range of unstable values of $\theta$ increases with $B$ in the predicted way. The growth rates are quadratic with respect to $L$ in the vicinity of the $L=0$ axis. The shape of the unstable regions of the $\theta, L$ parameter space becomes complicated for values of $L$ larger than 2 . In this region of parameter space, waves that are very slanted with respect to the meridional direction can be unstable to very short-wavelength zonal perturbation. 

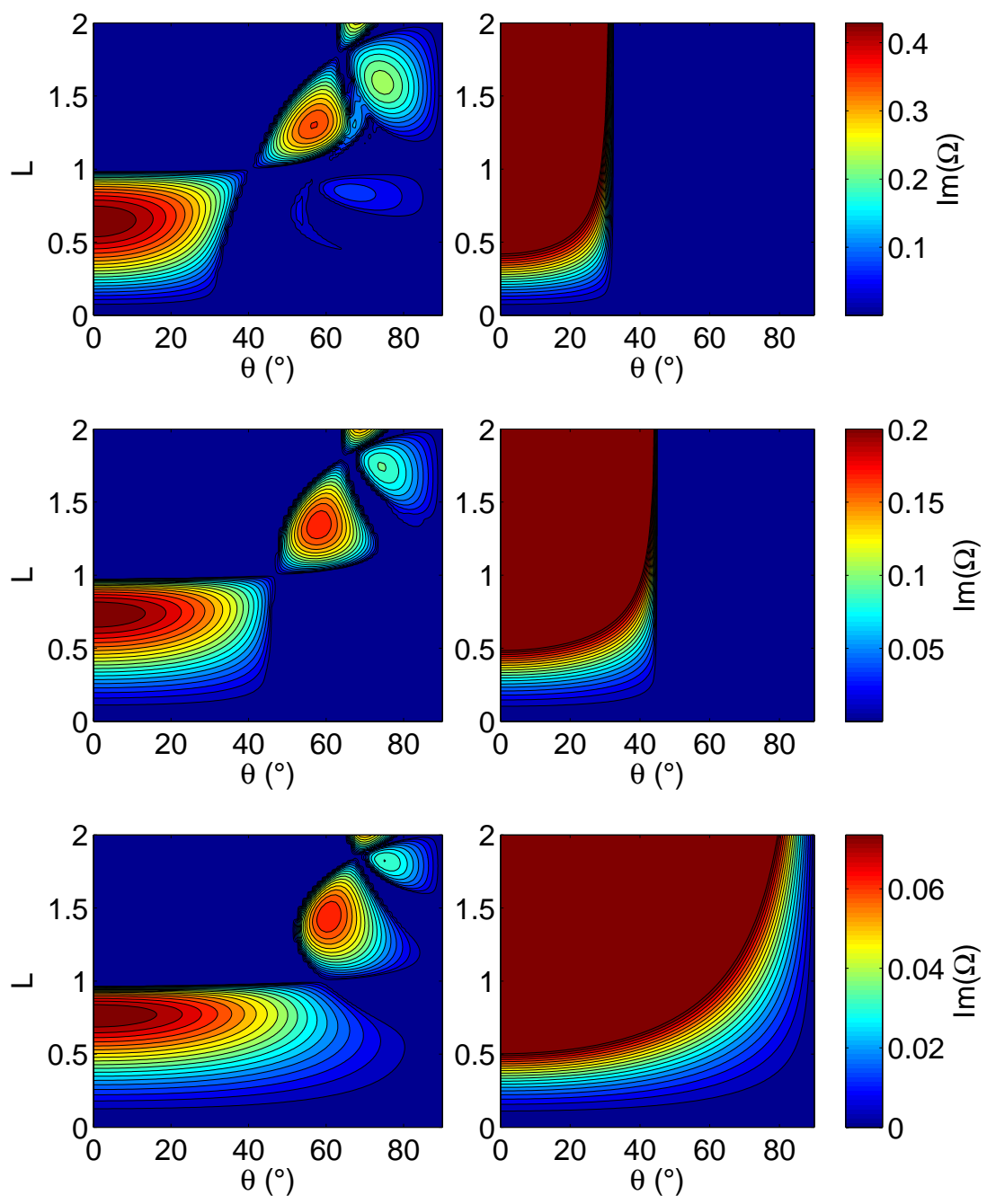

Fig. 7. Growth rates obtained for fixed non-linearity parameter $M=2$, as a function of the angle $\theta$ and the perturbation meridional wavenumber $L$, for $B=0.1$ (top), $B=1$ (center), $B=4$ (bottom). Left: Floquet numerical code. Right: Equation (18).

Equation (18) obviously fails at capturing these details. Finally, a general remark is that the system behaves regularly in the vicinity of the $\theta=0^{\circ}$ axis, and that the results displayed in Fig. 6 were indeed representative.

\section{Discussion}

We have mentioned above the failure of the barotropic mechanism to explain the instability in the stratified case. The argument fails because in the stratified case the zonal flow perturbation reacts via the vortex stretching component of its potential vorticity, which is absent from arguments based on linear momentum considerations. Figure 8 sketches a possible replacement based on potential vorticity conservation arguments and thus likely to be valid in more realistic settings.

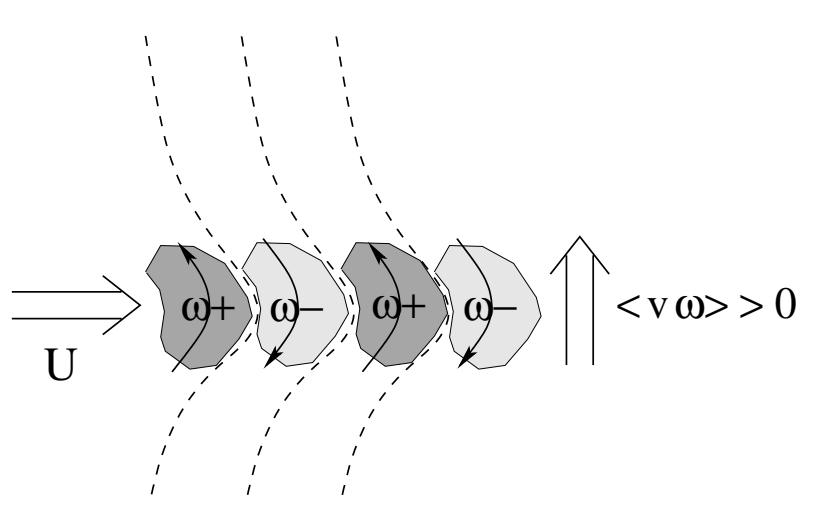

Fig. 8. Sketch of the stratified Rossby wave instability mechanism. $U$ represents the zonal flow distribution. The dashed lines represent the wave fronts, and the full arrows represent the associated flow velocities. Relative vorticity anomalies are marked with grey patches. 
As in the original argument of Sect. 1, a zonal velocity anomaly distorts the wavefronts of a base wave. Vorticity is present in the undisturbed wave along the surfaces separating northward and southward velocities. This vorticity is in quadrature with the meridional velocities, and the zonal average of its transport vanishes. The relative vorticity perturbation due to the wavefronts distortion, in contrast, is in phase with the meridional velocities (northward-going particles have to turn to their left, and thus possess cyclonic vorticity as well as northward velocity), and a non-zero meridional flux thus occurs. Relative vorticity flows northward across an eastward jet, as apparent on the graph. In a homogeneous setting, the zonal flow will become anticyclonic northward of the jet, and cyclonic southward. The cusp-like shape of the zonal velocity profile will strengthen, and we recover the original argument. In a stratified setting, for jet length scales much larger than the Rossby radius of deformation, the flow of relative vorticity can significantly force the vortex stretching term of the zonal flow, and tends to "pump" streamfunction to the right of the jet downstream direction, thereby strengthening it. We see that the interaction of a Rossby wave with a zonal jet is an efficient and straightforward way of generating a counter-gradient flow of potential vorticity across the jet.

\section{Conclusions}

The conclusions we can draw at the end of this study are of two different natures.

From a rather technical point of view, we have developed and validated a phase dynamical approach to the problem of the evolution of a finite-amplitude Rossby wave on the $\beta$-plane and its interaction with an infinitesimal zonal flow. The predictions have been quantitatively tested against numerical linear stability codes for the one base wave, zonal perturbations, inviscid, particular case of the instability studied by (Lorenz, 1972; Gill, 1974; Sivashinsky, 1985; Manfroi and Young, 2002; Lee and Smith, 2003). The approach has then been extended to the simple case of the reduced-gravity quasi-geostrophic model, and the differences between the two settings have been discussed. Indications of the difficulties to be expected in the extension of the method to multiplebaroclinic modes dynamical frameworks have finally been given.

From a more scientific point of view, we have provided accurate approximate expressions for the growth rates of the instability in both settings. The introduction of an intrinsic length scale, the Rossby radius of deformation, to the problem, has been shown not to modify significantly the scale selection properties of the instability, the most unstable length scale for the zonal perturbations remaining essentially marginally higher than the base flow wavelength. The growth rates have however been showed to be significantly affected, a decrease in deformation radius being associated to a de- crease in perturbation growth rate. Stratification also has the effect of introducing a threshold in base wave amplitude for instability, and to broaden the unstable wavevector direction range. Approximate analytical expressions for these different features have been provided. Finally, the classical physical interpretation of the instability has been extended to the case of a simple stratified medium.

The simple flow situation studied here needs to be generalized in many ways:

In particular, the results presented here have been obtained in the very specific case of one single base wave. Other results, not reported here, have been obtained in the two-layer quasi-geostrophic model setting, but the waves in the two baroclinic modes had to possess colinear wavevectors. A closer approach to reality, however, would require the analysis of a population of waves in statistical equilibrium and of their interaction with the zonal velocity profile. Whether such a study could benefit from the insight provided by the phase dynamics framework is not known.

We have only considered zonal perturbations to the base wave. This is justified by our focus on the instability as a feeding mechanism for zonal jets. Considering non-zonal perturbations in the phase dynamics framework is feasible, but the very convenient $\Omega=0$ solution does not exist in this problem, and obtaining the growth rate estimates requires solving a cubic, which leads to cumbersome expressions. One of the referees remarked that our discussion of the physical mechanism makes no explicit mention of $\beta$, whose role in the instability is thus unclear. Indeed, setting $\beta$ to 0 does not change the dimensional growth rate of the instability in the special case of zonal perturbations growing on a base wave with $\theta$ close to 0 . For $\theta \neq 0$ and/or non-zonal perturbations, the $\beta$-effect however plays a clear "detuning" role, in that it induces propagation of the phase and amplitude perturbations at the group velocity associated with the base flow wave, and of the $U$ perturbation at the (usually very different) phase velocity associated with its wavelength. Gaining a better understanding of the role of $\beta$ in the angular selection of base waves and unstable perturbations could help understand why the jets are actually zonal.

An interesting extension of the phase dynamical theory would be to modify it to accommodate slowly growing waves, such as for instance baroclinic waves close to the onset of instability. A possible outcome could be a theory of the complete chain going from an unstable mean flow, uniform in the meridional direction, to baroclinic waves, and to the zonal jets they feed in turn. Such a theory would be a very rich source of new insight.

In numerical simulations of the ocean (Nakano and Hasumi, 2005) as well as in observations (Maximenko et al., 2008), the jets usually appear as rather faint features superimposed on a sea of coherent vortices rather than waves. A possible direction for the future could be to make an analytical study, similar in spirit to the numerical work of (Dritschel 
and McIntyre, 2008), of the interaction of an isolated coherent vortex with an infinitesimal zonal flow perturbation.

\section{Appendix A}

\section{Floquet analysis of Eq. (1)}

Our aim in this appendix is to describe the Floquet code we have used to check and extend the results obtained through the phase dynamics analysis developed in the main text. After a description of the general problem settings, we present the algebraic system obtained through Fourier expansion of the Floquet eigenmodes.

\section{A1 Problem formulation}

The basic equation of motion is Eq. (1), which we repeat here for completeness:

$$
\partial_{t} \Delta \psi+\partial_{x} \psi+M J(\psi, \Delta \psi)=0
$$

We now decompose $\psi$ into a base flow streamfunction and a perturbation:

$\psi=\psi_{0}+f$

with

$\psi_{0}=\sin (\cos (\theta)(x+t)+\sin (\theta) y)$

Introducing this decomposition in Eq. (1), using the fact that $\psi_{0}$ alone is one of its solutions, and keeping only terms linear in the perturbation streamfunction, we obtain that the perturbation evolves according to:

$\partial_{t} \Delta f+\partial_{x} f+M J\left(\psi_{0}, \Delta f\right)+M J\left(f, \Delta \psi_{0}\right)=0$.

Performing the change of variable $x \rightarrow x+t$, we change to a frame of reference in which the base flow pattern is stationary. In this frame of reference, and using the fact that $\Delta \psi_{0}=-\psi_{0}$, the equation of motion reads

$\partial_{t} \Delta f+\partial_{x}(\Delta f+f)+M J\left(\psi_{0}, \Delta f+f\right)=0$,

with $\psi_{0}$ now time-invariant

$\psi_{0}=\sin (\cos (\theta) x+\sin (\theta) y)$.

At this point, Floquet theory tells us that the eigenmodes of Eq. (A2) are of the form:

$f=e^{i[K x+L y-\omega t]} \widehat{f}(x, y)$

where $\widehat{f}$ is a function which has the same wavelengths in the $\mathrm{x}$ - and $\mathrm{y}$-directions as the base flow Rossby wave, but can have a more complicated pattern.

\section{A2 Fourier expansion}

To make progress in the study of Eq. (A2), we introduce for $\widehat{f}$ a Fourier decomposition in $x$ and $y$.

$f=e^{i[K x+L y-\omega t]} \sum_{n=-\infty}^{\infty} h_{n} e^{i n[\cos (\theta) x+\sin (\theta) y]}$

Replacing $f$ in Eq. (A2) by its expression (A3), performing the necessary differentiations, and collecting the resulting terms according to their spatial wavenumber, we obtain the algebraic set of equations linking the amplitudes $\left(h_{n}\right)_{n \in \mathbb{Z}}$. Defining for $n \in \mathbb{Z}$

$l_{n}=n^{2}+2 n[L \sin (\theta)+K \cos (\theta)]+L^{2}+K^{2}$,

we obtain the set of equations as:

$\forall n \in \mathbb{Z}, 0=\left[[n \cos (\theta)+K]\left[l_{n}-1\right]-\omega l_{n}\right] h_{n}$

$$
\begin{aligned}
& +\frac{M}{2}[\cos (\theta) L-\sin (\theta) K]\left[l_{n+1}-1\right] h_{n+1} \\
& +\frac{M}{2}[\cos (\theta) L-\sin (\theta) K]\left[l_{n-1}-1\right] h_{n-1}
\end{aligned}
$$

After truncation of the system to $n \in\{-N \ldots N\}$ for some integer $N$, this set of equations can easily be solved numerically for $\omega$ as an algebraic eigenproblem. The results shown in the main text have been obtained with $N=16$ (with the exception of Fig. 4c, which shows that $N=1$ actually gives a very good approximation of the result in the barotropic case).

\section{Appendix B}

\section{Floquet analysis of Eq. (14)}

This section is complementary to the previous one, and presents the Floquet analysis of Eq. (14):

$\partial_{t}[\Delta-B] \psi+(B+1) \partial_{x} \psi+M J(\psi, \Delta \psi)=0$.

$\psi$ is decomposed into a base flow, $\psi_{0}$, and a perturbation, $f$, such that:

$\psi=\psi_{0}+f, \psi_{0}=\sin (\cos (\theta)(x+t)+\sin (\theta) y)$.

Introducing this decomposition in Eq. (14), using the fact that $\psi_{0}$ alone is one of its solutions, using the fact that $\Delta \psi_{0}=-\psi_{0}$, keeping only terms linear in the perturbation streamfunction, and finally using the skew-symmetry of the Jacobian with respect to its arguments, we obtain that the perturbation evolves according to:

$\partial_{t}[\Delta-B] f+(B+1) \partial_{x} f+M J\left(\psi_{0},[\Delta+1] f\right)=0$.

The change of variable $x \rightarrow x+t$ makes the base flow pattern stationary, and changes the equation to:

$\partial_{t}[\Delta f-B f]+\partial_{x}(\Delta f+f)+M J\left(\psi_{0}, \Delta f+f\right)=0$, 
with

$\psi_{0}=\sin (\cos (\theta) x+\sin (\theta) y)$.

Once again, we remark that, once the proper scalings and translations have been performed, the only difference between the problems in the barotropic and reduced-gravity cases lies is the fact that in the later case the vortex stretching term is the dominant variable at the slow $Y$-dependence scale for non-zero $B$. Introducing again the expansion

$f=e^{i[K x+L y-\omega t]} \sum_{n=-\infty}^{\infty} h_{n} e^{i n[\cos (\theta) x+\sin (\theta) y]}$,

we obtain the set of algebraic equations for $\omega$ and the $\left(h_{n}\right)_{n \in \mathbb{Z}}$ :

$\forall n \in \mathbb{Z}, 0=\left[[n \cos (\theta)+K]\left[l_{n}-1\right]-\omega\left[l_{n}+B\right]\right] h_{n}$

$$
\begin{aligned}
& +\frac{M}{2}[\cos (\theta) L-\sin (\theta) K]\left[l_{n+1}-1\right] h_{n+1} \\
& +\frac{M}{2}[\cos (\theta) L-\sin (\theta) K]\left[l_{n-1}-1\right] h_{n-1}
\end{aligned}
$$

Again, this system is solved numerically as an algebraic eigenproblem after truncation to $n \in\{-N \cdots N\}$, with $N=16$.

\section{Appendix C}

\section{The phase dynamics approach as} an approximation of the truncated Floquet analysis $(\mathbf{n} \in\{-\mathbf{1}, \mathbf{0}, \mathbf{1}\})$

One referee raised the issue of the relations between the different approximations schemes, and most importantly between the Floquet analysis, truncated to $(n \in\{-1,0,1\})$, which is well known to provide very good analytic growth rate estimates, and the phase dynamics approach, on which the present study is based. This section is devoted to a detailed investigation of this.

Perturbing the base flow streamfunction of Eq. (A1) with amplitude, phase and zonal flow perturbations of the kind used in the phase dynamics study, one obtains

$\psi(x, y, t)$

$=\varepsilon \Psi(y, t)+\operatorname{Im}\left(e^{i[\cos (\theta) x+\sin (\theta) y+\varepsilon \phi(y, t)+i \varepsilon \chi(y, t)]}\right)$

Introducing for $\Psi, \phi, \chi$ their usual sinusoidal dependencies in $y$ and $t$ :

$$
\left|\begin{array}{l}
\phi \\
\chi \\
\Psi
\end{array}=e^{i[L y-\omega t]}\right| \begin{aligned}
& \widehat{\phi} \\
& \widehat{\chi} \\
& \widetilde{\Psi}
\end{aligned},
$$

one obtains after some algebra the perturbed streamfunction as:

$$
\begin{aligned}
\psi(x, y, t)= & \psi_{0}(x, y, t) \\
& +\varepsilon e^{i(L y-\omega t)}[\widehat{\Psi} \\
& +\frac{1}{2} e^{i[\cos (\theta) x+\sin (\theta) y]}(\widehat{\phi}+i \widehat{\chi}) \\
& \left.+\frac{1}{2} e^{-i[\cos (\theta) x+\sin (\theta) y]}(\widehat{\phi}-i \widehat{\chi})\right] .
\end{aligned}
$$

This expression can be recognized as the Floquet-Fourier expansion (A3), restricted to the $K=0$ case, and to its $n \in$ $\{-1,0,1\}$ members, if the following identifications are made:

$$
\begin{aligned}
h_{-1} & =\frac{\varepsilon}{2}(\widehat{\phi}-i \widehat{\chi}) \\
h_{0} & =\varepsilon \widehat{\Psi} \\
h_{1} & =\frac{\varepsilon}{2}(\widehat{\phi}+i \widehat{\chi})
\end{aligned}
$$

A straightforward mapping thus exists between the perturbation types handled by the two methods of approximation. The Floquet-Fourier procedure, however, makes no assumption about the slowness of the perturbations variations in the meriodional directions. The equations of motion for $h_{-1}$, $h_{0}$ and $h_{1}$ thus suffer no restrictions for large meridional wavenumber $L$.

Replacing $h_{0}, h_{-1}$ and $h_{1}$ by their expressions in the Floquet-Fourier equations of motion (A4), developing, and using the correspondence $-i \omega \leftrightarrow \partial_{t}, i L \leftrightarrow \partial_{x}$, one can obtain the following equations of motion for the phase dynamics variables in the barotropic setting:

$$
\begin{aligned}
\partial_{t} U= & \frac{M}{2}\left[\cos (\theta) \partial_{y y} \phi-\sin (2 \theta) \partial_{y} \chi\right] \\
\partial_{t}\left(1-\partial_{y y}\right) \phi+\sin (2 \theta) \partial_{y} \phi= & -2 \sin (\theta) \partial_{t y} \chi-\cos (\theta) \partial_{y y} \chi \\
& -M \cos (\theta)\left[\partial_{y y}+1\right] U \\
\partial_{t}\left(1-\partial_{y y}\right) \chi+\sin (2 \theta) \partial_{y} \chi= & 2 \sin (\theta) \partial_{t y} \phi+\cos (\theta) \partial_{y y} \phi .
\end{aligned}
$$

These equations are more complicated than those derived by the multiple-scales procedure discussed in the main text, but are valid without restriction on the variations of $\phi, \chi, U$ with respect to $y$. It is clear that they reduce to Eqs. (10) in the limit of slow variations.

The equations of motion in the reduced-gravity setting read:

$$
\begin{aligned}
\partial_{t}\left(B-\partial_{y y}\right) \Psi= & \frac{M}{2}\left[\cos (\theta) \partial_{y y y} \phi-\sin (2 \theta) \partial_{y y} \chi\right] \\
\partial_{t}\left(1+B-\partial_{y y}\right) \phi+\sin (2 \theta) \partial_{y} \phi= & -2 \sin (\theta) \partial_{t y} \chi-\cos (\theta) \partial_{y y} \chi \\
& +M \cos (\theta)\left[\partial_{y y y}+\partial_{y}\right] \Psi \\
\partial_{t}\left(1+B-\partial_{y y}\right) \chi+\sin (2 \theta) \partial_{y} \chi= & 2 \sin (\theta) \partial_{t y} \phi+\cos (\theta) \partial_{y y} \phi .
\end{aligned}
$$


These systems of equations remain approximations in the sense that they are only truncated counterparts of the full system (A4). As mentioned in the main text, the fact that the truncation yields good results is indicative of the fact that the instability mechanism relies primarily on phase and amplitude perturbations of the base flow waves, and that the changes in the waveform are essentially limited to the introduction of the zonal flow component. More subtle changes in the waveform, which would require higher order harmonics to be represented, do not seem to play a key role. This finding turns out to be valid also for non-zonal perturbations in the barotropic case, but its validity seems restricted to slow meridional variations in the reduced-gravity case (not shown).

Acknowledgements. It is a pleasure to acknowledge here stimulating discussions with M. Ben Jelloul, P. Cessi, A. Colin de Verdière, B.-L. Hua, S. Fauve, B. Le Cann and W. R. Young. One referee made very constructive comments that lead to substantial improvements of the manuscript.

Edited by: S. Wiggins

Reviewed by: two anonymous referees

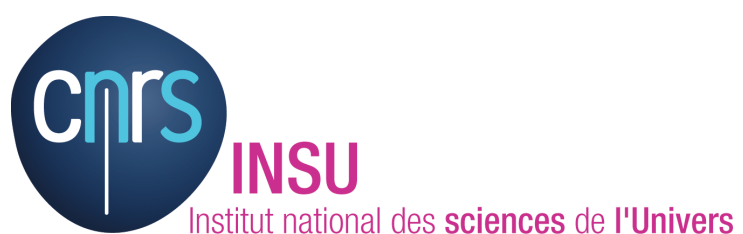

The publication of this article is financed by CNRS-INSU.

\section{References}

Berloff, P., Kamenkovich, I., and Pedlosky, J.: A mechanism of formation of multiple zonal jets in the oceans, J. Fluid Mech., 628, 395-425, 2009.

Dritschel, D. G. and McIntyre, M. E.: Multiple jets as PV staircases: the Phillips effect and the resilience of eddy-transport barriers, J. Atmos. Sci., 65, 855-874, 2008.

Galperin, B. H., Nakano, H., Huang, H., and Sukoriansky, S.: The ubiquitous zonal jets in the atmospheres of giant planets and Earth's oceans, Geophys. Res. Lett., 21, L13303, doi:10.1029/2004GL01969, 2004.

Gill, A. E.: The stability of planetary waves on an infinite $\beta$-plane, Geophys. Astro. Fluid, 6, 29-47, 1974.

Lee, Y. and Smith, L. M.: Stability of Rossby waves in the $\beta$-plane approximation, Physica D, 179, 53-91, 2003.

Lorenz, E. N.: Barotropic instability of Rossby wave motion, J. Atmos. Sci., 29, 258-264, 1972.

Manfroi, A. J. and Young, W. R.: Slow evolution of zonal jets on the $\beta$-plane, J. Atmos. Sci., 56, 784-800, 1999.

Manfroi, A. J. and Young, W. R.: Stability of $\beta$-plane Kolmogorov flow, Physica D, 162, 208-232, 2002.

Maximenko, N. A., Melnichenko, O. V., Pearn, N. P., and Hideharu, S.: Stationary mesoscale jet-like features in the ocean, Geophys. Res. Lett., 35, L08603, doi:10.1029/2008GL033267, 2008.

Nakano, H. and Hasumi, H.: A series of zonal jets embedded in the broad zonal flows in the Pacific obtained in eddy-permitting ocean general circulation models, J. Phys. Oceanogr., 35, 474488, 2005.

Panetta, L.: Zonal jets in wide baroclinically unstable regions: Persistence and scale selection, J. Atmos. Sci., 50, 2073-2106, 1993.

Rhines, P. B.: The dynamics of unsteady currents, chap. 7, 189-318, John Wiley and Sons, Inc., New York, 1977.

Rhines, P. B.: Waves and turbulence on a beta-plane, J. Fluid Mech., 69, 417-443, 1975.

Sivashinsky, G. I.: Weak turbulence in periodic flows, Physica D, 17, 243-255, 1985.

Thomson, A. and Young, W. R.: Baroclinic eddy heat fluxes: zonal flows and energy balance, J. Atmos. Sci., 64, 3214-3231, 2007.

Vallis, G. K.: Atmospheric and Oceanic Fluid Dynamics, Cambridge University Press, Cambridge, UK, 2006.

Vallis, G. K. and Maltrud, M.: Generation of mean flows and jets on a beta plane and over topography, J. Phys. Oceanogr., 23, 13461362, 1993.

Young, W. R. and Rhines, P. B.: Rossby wave action, enstrophy and energy in forced mean flows, Geophys. Astro. Fluid, 15, 39-52, 1980. 